\title{
Effect of Sagittal Dentoskeletal Discrepancies on the Vermilion Height and Lip Area
}

\author{
Arun Joseph, Shobha Sundareswaran, Sandhya Srinivas
}

Department of Orthodontics and Dentofacial Orthopedics, Government Dental College (Kerala University of Health Sciences), Calicut, Kerala, India

Cite this article as: Joseph A, Sundareswaran S, Srinivas S. Effect of Sagittal Dentoskeletal Discrepancies on the Vermilion Height and Lip Area. Turk J Orthod 2019; 32(2): 79-86

\begin{abstract}
Objective: A frontal evaluation of the lips could provide important information during a routine clinical evaluation of facial aesthetics. There is a lack of ample evidence in the literature regarding variations in the vermilion height and lip area in various sagittal discrepancies when assessing facial aesthetics. The aim of this cross-sectional study was to evaluate and compare the vermilion height and lip area in dentoskeletal Class I, Class II, and Class III malocclusions.
\end{abstract}

Methods: Subjects included female patients divided into four groups (Angle's Class I bimaxillary proclination [Class I BMP], Class II Division I [Class II Div 1], Class III and Class I normal [Class I N]) with 36 samples each. Standardized frontal facial photographs were taken at rest and during a posed smile. Thirty-five landmarks on the upper and lower lips were identified for measurements of the vermilion height and lip area. A one-way analysis of variance was used to identify overall differences, and the post-hoc Bonferroni test was applied for multiple comparisons.

Results: Class III showed a significantly smaller upper-lip area and significantly higher ratios of the upper-to-lower lip vermilion height/area. The ratios displayed an increasing trend from the midline to the corners of the mouth. Class I BMP and Class II Div 1 had significantly larger upper and lower-lip areas.

Conclusion: Morphology of the lips is significantly correlated with underlying anteroposterior dentoskeletal discrepancies. During a clinical examination, a critical frontal evaluation of the lips is important as it is apparently indicative of the underlying sagittal discrepancy, especially in skeletal Class III malocclusions.

Keywords: Sagittal discrepancy, lip area, lip proportion, photographic analysis, vermilion height

\section{INTRODUCTION}

The face plays a key role in communication and interaction, involving all social relationships among human beings (1). Facial aesthetics is not only one of the most important motives for patients seeking orthodontic treatment, but also a vital objective for the orthodontist (2). The lips and teeth are considered fundamental factors in facial appearance (3). Majority of studies have evaluated facial aesthetics in profile view $(3,4)$. However, patients tend to judge facial appearances by assessing their frontal view in the mirror (5). Hence studies investigating lip aesthetics in the frontal view are warranted.

More prominent, larger-than-average-size lips have been reported in attractive adolescents (6). The vertical thickness of the lips has been reported to be the most important component of a pleasant smile by both orthodontists and lay persons (7). They concluded that while the vertical thickness of the lower lip was an aesthetic determinant for laypersons, the vertical thickness of the upper lip was an aesthetic determinant for both laypersons and orthodontists. The last two decades of the $20^{\text {th }}$ century showed a trend toward fuller lips among Caucasian female models, closer to the African-Americans (8). Obviously, the vertical lip thickness is an important factor in the determination of attractiveness of the mouth (9). 
The position of the lips is closely related to the teeth and alveolar processes (8). A previous study has reported the upper lip to be correlated positively with the position of maxillary incisors; the more protrusive the incisors, the fuller the upper lip (7). The orthodontic retraction of anterior teeth following four premolar extractions has been shown to significantly decrease the vermilion height and lip area in bimaxillary protrusive patients (5). This obviously implies that the morphology of lips in frontal view is affected by the underlying hard tissue anteroposterior discrepancies. Unfortunately, there is a lack of ample evidence in the literature regarding variations in the vermilion height and lip area in various sagittal discrepancies while assessing facial aesthetics. How do anteroposterior dentoskeletal discrepancies like Class I, Class II, and Class III affect frontal lip morphology? This lacuna in current knowledge has not been addressed so far. Hence, the aim of the present study was to evaluate the lip morphological characteristics in dentoskeletal Class I, Class II, and Class III malocclusions in a population of Dravidian ethnic origin.

\section{METHODS}

Approval for the study was obtained from the Institutional Ethics Committee (IEC no: 45/2014/DCC). Subjects were chosen from patients reporting to the Department of Orthodontics and comprised of adult females of Dravidian ethnicity. They were divided into four groups based on the following criteria: Class I BMP-Angle's Class I malocclusion with bimaxillary proclination: an ANB angle of $1^{\circ}-3^{\circ}$, the Wits appraisal 0 to $-3 \mathrm{~mm}$, an interincisal angle less than $125^{\circ}$, Angle's Class I molar relationship, profile showing circumoral convexity with both the lips positioned beyond Ricketts aesthetic plane (10); Class II Div 1-Angle's Class II Division 1 malocclusion: an angle ANB $>4^{\circ}$, the Wits appraisal greater than $-1 \mathrm{~mm}$, an overjet more than $5 \mathrm{~mm}$, Angle's Class II molar relationship, clinically retrognathic, Class
II profile (10); Class III-Angle's Class III malocclusion: an ANB angle lesser than $1^{\circ}$, the Wits appraisal less $\leq-4 \mathrm{~mm}$, Angle's Class III molar relationship, clinically prognathic Class III profile (10); Class I N-Angle's Class I normal occlusion: An angle ANB of $1^{\circ}-3^{\circ}$, the Wits appraisal 0 to $-3 \mathrm{~mm}$, an interincisal angle of $130^{\circ}-135^{\circ}$, Angle's Class I molar relationship, clinically orthognathic Class I profile (10). Patients with previous orthodontic treatment, facial asymmetries, or craniofacial anomalies, short upper lip, severe crowding, spacing, incisor displacements, subdivisions (unilateral), transverse discrepancies, impacted tooth, and partial anodontia were excluded from the study. For a minimum difference of $2 \mathrm{~mm}$ and a standard deviation of 3 , (11) the sample size was deemed to be 36 in each group for obtaining a meaningful significance.

Informed written consent from the parents and the patients was obtained. A review of the literature showed that significant sexual dimorphism exists in the lip morphology (12-14). Female lips are reported to thicken till the age of 14 years, after which they remain the same $(15,16)$. Therefore, only female subjects aged 18-25 years (mean age \pm standard deviation: $20.6 \pm 2.1$ years for Class I BMP; $19.5 \pm 1.8$ years for Class II Div $1 ; 21.3 \pm 3.2$ years for Class III; and 20.1 \pm 1.2 years for Class I N) were included.

The frontal photographs were taken at rest and during a posed smile in a normal standing posture with the head fixed by ear rods, with a distance of $1.5 \mathrm{~m}$ between the camera lens (SONY DSC-HX400V) and the subject. All photographs were taken by zooming the lens to $10 x$ magnification. The subjects were not allowed to wear any facial cosmetics/make-up. The midsagittal plane of the head was aligned with the center of the camera lens using a tripod stand. To obtain the rest position, subjects were made to stand straight in front of the camera, keeping the ala tragus line parallel to the floor. A custom-made head-holding device with a stand was constructed for this purpose.

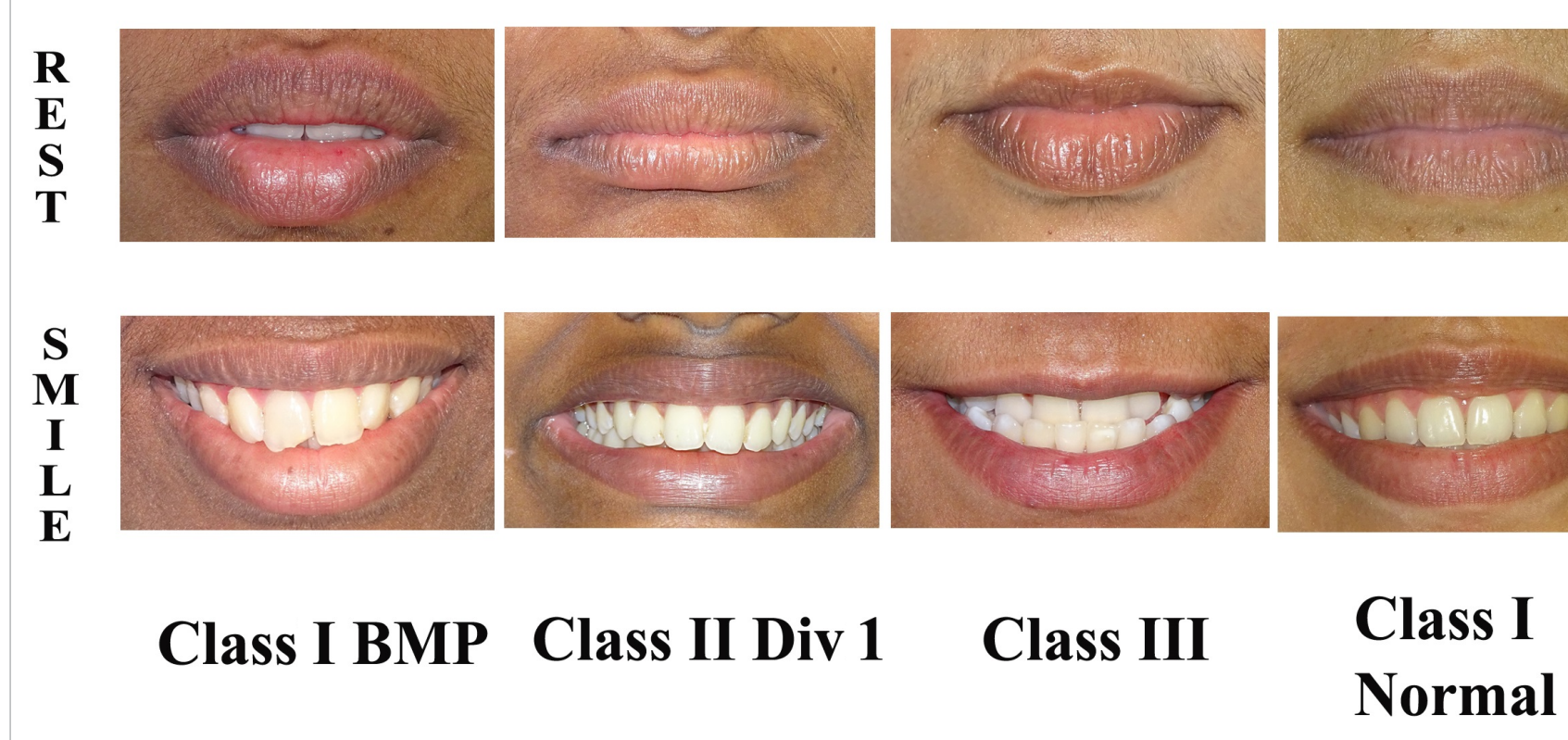


While being photographed, the subjects were asked to keep their teeth slightly apart, and the perioral soft tissues and mandibular posture unstrained at rest. Subjects were asked to say "Mississippi" and then keep the lips in that position. Each subject was coached and asked to achieve the same lip position at least twice in succession before a photograph was taken. To achieve the smiling position, subjects were asked to fully smile and say "cheese" (11), and reproduce the same smile at least twice successively. The subjects easily attained a reproducible maximum smile, and photographs were taken in this position (Figure 1).

All frontal photographs (size $14.81 \mathrm{~W} \times 9.87 \mathrm{H}$ inches and resolution 350 pixels/inch) were copied to the Adobe Photoshop CS3 Extended (version 10.0, Adobe systems 1990), and the lip outlines were drawn and lips shaded. After drawing the vertical and horizontal lines, 35 landmarks were marked as shown in Figure 2. For this purpose, an $X$-axis was drawn parallel to the line connecting the right and left irises through the subnasale point (Sn), whereas the $Y$-axis was drawn perpendicular to the $X$-axis through the Sn point. Two vertical lines were then drawn through the right and left superior vermilion points $(9,11)$. Both the right and left sides were divided into three equal parts from the superior vermilion point of the lip to the corners of the mouth $(6,14)$. Four more vertical lines were drawn through landmarks numbered 7 ,
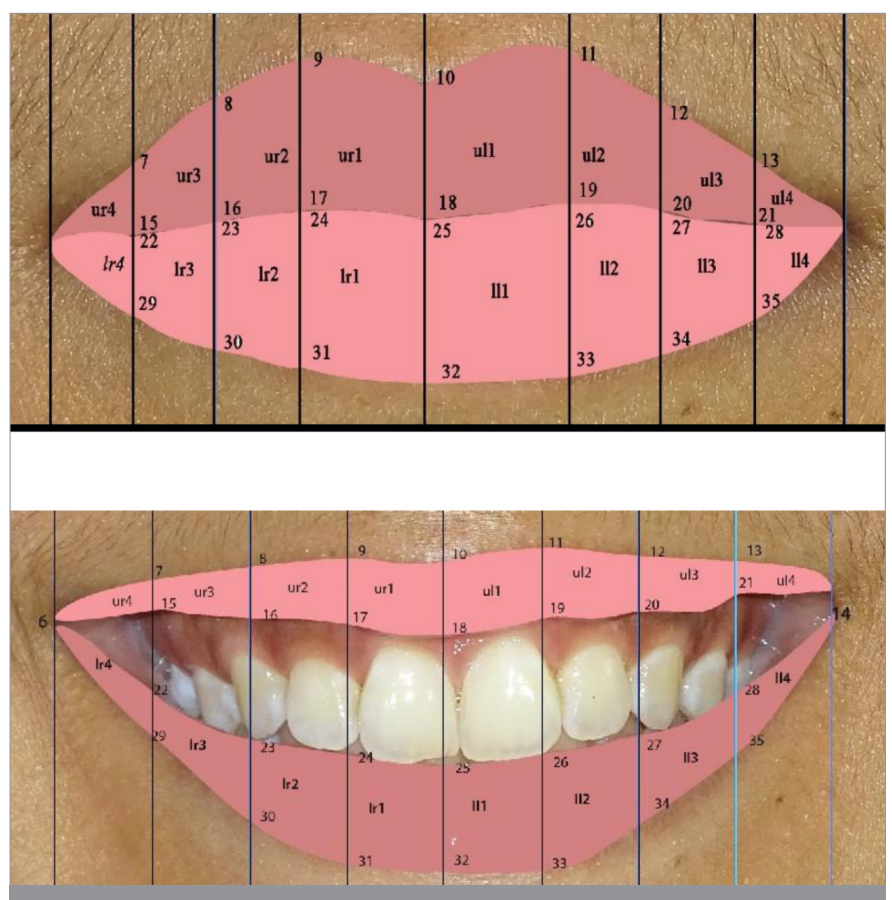

Figure 2. Lips at rest and during smile with landmarks
8,12 , and 13. The landmarks numbered 6-14 and 15-21 were allocated for the upper lip, and 22-28 and 29-35 were allocated for the lower lip. The vermilion height $(7-15,8-16,9-17,10-18$, 11-19, 12-20, 13-21, 22-29, 23-30, 24-31, 25-32, 26-33, 27-34, and 28-35) and the lip area of both lips was measured using Adobe Photoshop CS3 Extended (version 10.0). Details about the landmarks have been reported earlier $(5,11)$. To compare the facial size, the distance between the right and left irises of the patient and the control groups were measured. Post-hoc Bonferroni tests confirmed that there were no significant differences in facial size among the four groups. There were no significant variations in the age range either among the groups.

Statistical analysis: All the data were statistically evaluated with the SPSS software (SPSS Inc. Released 2009. PASW statistics for Windows, Version 18, Chicago, IL). The normality of the data was tested using the Koglomorov-Smirnov test. All the variables followed normal distribution. One-way analysis of variance (ANOVA) was used to identify overall differences in mean values of vertical vermilion ratios and lip-area ratios in the four groups. The level of significance was set at $p<0.05$. When differences between groups were found to be significant, the post-hoc Bonferroni test for multiple comparisons was applied. To test intra-observer reliability, ten photographs of each sample were traced and digitized on two separate occasions, three weeks apart. All intraclass correlation coefficients for the vermilion height and lip-area measurements were greater than or equal to 0.88 , signifying a negligible error.

\section{RESULTS}

Table 1 shows the mean and standard deviation of the upper and lower total lip area with ANOVA, and Table 2 depicts multiple comparisons of the above among the four groups at rest and during smile. The upper lip total area in Class III was found to be lesser by $11 \mathrm{~cm}^{2}$ than the Class I BMP, by $9 \mathrm{~cm}^{2}$ than Class II Div 1 , and by $5 \mathrm{~cm}^{2}$ than Class I $\mathrm{N}$ group. These differences were found to be highly significant $(p<0.001)$. Similar highly significant differences were also apparent during smile.

A comparison of the upper and lower segment vermilion heights at rest and during smile is given in Figure 3. This graphic representation shows a consistent decrease in the vermilion height of the upper lip in Class III malocclusions. This is obvious across all segments of the upper lip during rest as well as smile.

The Class I BMP and Class II Div 1 groups displayed significantly higher values for both upper and lower total lip areas when com-

Table 1. Mean and standard deviation values for upper and lower total lip areas at rest and during smile with analysis of variance (ANOVA)

\begin{tabular}{|c|c|c|c|c|c|c|}
\hline & \multirow[b]{2}{*}{ Class I BMP $\left(\mathrm{cm}^{2}\right)$} & \multirow[b]{2}{*}{ Class II Div $1\left(\mathrm{~cm}^{2}\right)$} & \multirow[b]{2}{*}{ Class III $\left(\mathrm{cm}^{2}\right)$} & \multirow[b]{2}{*}{ Class I N ( $\left.\mathrm{cm}^{2}\right)$} & \multicolumn{2}{|c|}{ ANOVA } \\
\hline & & & & & $\mathbf{F}$ & sig \\
\hline ruta & $22.61 \pm 3.97$ & $20.83 \pm 3.36$ & $11.52 \pm 3.62$ & $16.48 \pm 3.27$ & 69.141 & 0.000 \\
\hline suta & $24.27 \pm 3.21$ & $22.71 \pm 3.37$ & $12.20 \pm 4.24$ & $17.57 \pm 3.57$ & 82.081 & 0.000 \\
\hline slta & $33.29 \pm 4.54$ & $30.68 \pm 5.02$ & $28.82 \pm 6.68$ & $25.97 \pm 4.50$ & 12.365 & 0.000 \\
\hline
\end{tabular}


Table 2. Multiple comparisons of upper and lower total lip areas at rest and during smile

\section{Dependent \\ Variable}

Rest upper

total area

(ruta)

Multiple Comparison

Class I BMP

\section{Class II D1}

Class III

Class I N

Class IID1

Class I BMP

Class III

Class I N

Class III

Class I BMP

Class II DI

Class I N

Class I N

Class I BMP

Class II D1

Class III

Rest lower

total area

(rlta)

\begin{tabular}{|c|c|c|c|}
\hline & Class II D1 & Class I BMP & 0.140 \\
\hline & & Class III & 0.432 \\
\hline & & Class I N & 0.000 \\
\hline & Class III & Class I BMP & 0.000 \\
\hline & & Class II DI & 0.432 \\
\hline & & Class I N & 0.146 \\
\hline & Class I controls & Class I BMP & 0.000 \\
\hline & & Class II D1 & 0.000 \\
\hline & & Class III & 0.146 \\
\hline Smile upper & Class I BMP & Class II D1 & 0.420 \\
\hline $\begin{array}{l}\text { total area } \\
\text { (suta) }\end{array}$ & & Class III & 0.000 \\
\hline & & Class I N & 0.000 \\
\hline & Class II D1 & Class I BMP & 0.420 \\
\hline & & Class III & 0.000 \\
\hline & & Class I N & 0.000 \\
\hline & Class III & Class I BMP & 0.000 \\
\hline & & Class II DI & 0.000 \\
\hline & & Class I N & 0.000 \\
\hline & Class I N & Class I BMP & 0.000 \\
\hline & & Class II D1 & 0.000 \\
\hline & & Class III & 0.000 \\
\hline Smile lower & Class I BMP & Class II D1 & 0.222 \\
\hline I area & & Class III & 0.003 \\
\hline & & Class I N & 0.000 \\
\hline & Class II D1 & Class I BMP & 0.222 \\
\hline & & Class III & 0.816 \\
\hline & & Class I N & 0.001 \\
\hline & Class III & Class I BMP & 0.003 \\
\hline & & Class II DI & 0.816 \\
\hline & & Class I N & 0.139 \\
\hline & Class I N & Class I BMP & 0.000 \\
\hline & & Class II D1 & 0.001 \\
\hline & & Class III & 0.139 \\
\hline
\end{tabular}

pared to Class III and Class I N groups at rest and during smile $(p<0.001)$. However, no statistically significant differences were observed between these two (Class I BMP and Class II Div 1).

The ratios of upper-to-lower lip vermilion height and area during rest and smile with ANOVA are given in Table 3. These ratios were obtained by dividing the lower-lip values by the corresponding upper ones with respect to both lip areas and vermilion heights at different points. This was done during rest and smile to yield the "rest vermilion ratio (rvr)," "smile vermilion ratio (svr)," "rest area ratio (rar)," "smile area ratio (sar)," "rest total area ratio (rtar)," and "smile total area ratio (star)". Results show highly significant differences $(p<0.001)$. A multiple comparison revealed that the skeletal Class III group was significantly different. The value of all the ratios in skeletal Class III were found to be significantly higher $(p<0.001)$. No significant variations were observed in the ratios for Class I BMP, Class II Div 1, and Class I controls. Figure 4 depicts the ratios of the upper-to-lower vermilion height and lip area at rest and during smile.

\section{DISCUSSION}

The importance of evaluating aesthetics in the frontal view has been well emphasized with an increasing number of orthodontists shifting their focus from the sagittal plane to the frontal, while evaluating patients for orthodontic treatment (17). The mouth being the center of communication in the face, the aesthetic appearance of the oral region during rest, speech, and smile is a conspicuous part of facial attractiveness (11).

Photographs provide a conventional documentation of the soft tissues of the face and are considered extremely reliable, as facial landmarks can be located consistently $(18,19)$. Though various soft tissue facial analyses based on standardized diagnostic photographs are available (18), none of them focus on morphology and proportions of the lips in frontal view. Whether lip frontal morphology and proportions are affected by underlying sagittal dentoalveolar discrepancies have thus far, to the best of our knowledge, not been evaluated. The present study focuses on the frontal evaluation and comparison of the upper- and lower-lip morphology in skeletal Class I BMP, Class II Div 1, Class III, and Class I N subjects.

Our investigation revealed statistically highly significant decrease in the upper-lip area of the skeletal Class III group, as compared to others at rest and during a posed smile. The decrease in the vermilion height of the upper lip in skeletal Class III is also obvious at rest and during smile (Figure 3). Clinically, this was observed to be a remarkable identifying feature during a regular extraoral examination of the face and lips. This is in contrast to the observations by Rafiqul Islam et al., who in an evaluation of the lip morphology of skeletal Class III cases following orthognathic surgery reported that the pre-treatment areas of the upper and lower lips in Class III were significantly larger than Class I controls (17).

The Class I BMP group in our study showed a statistically highly significant increase in both the upper and lower-lip areas and 
Table 3. Upper-to-lower-lip vermilion height and lip-area ratios during rest and smile with analysis of variance (ANOVA)

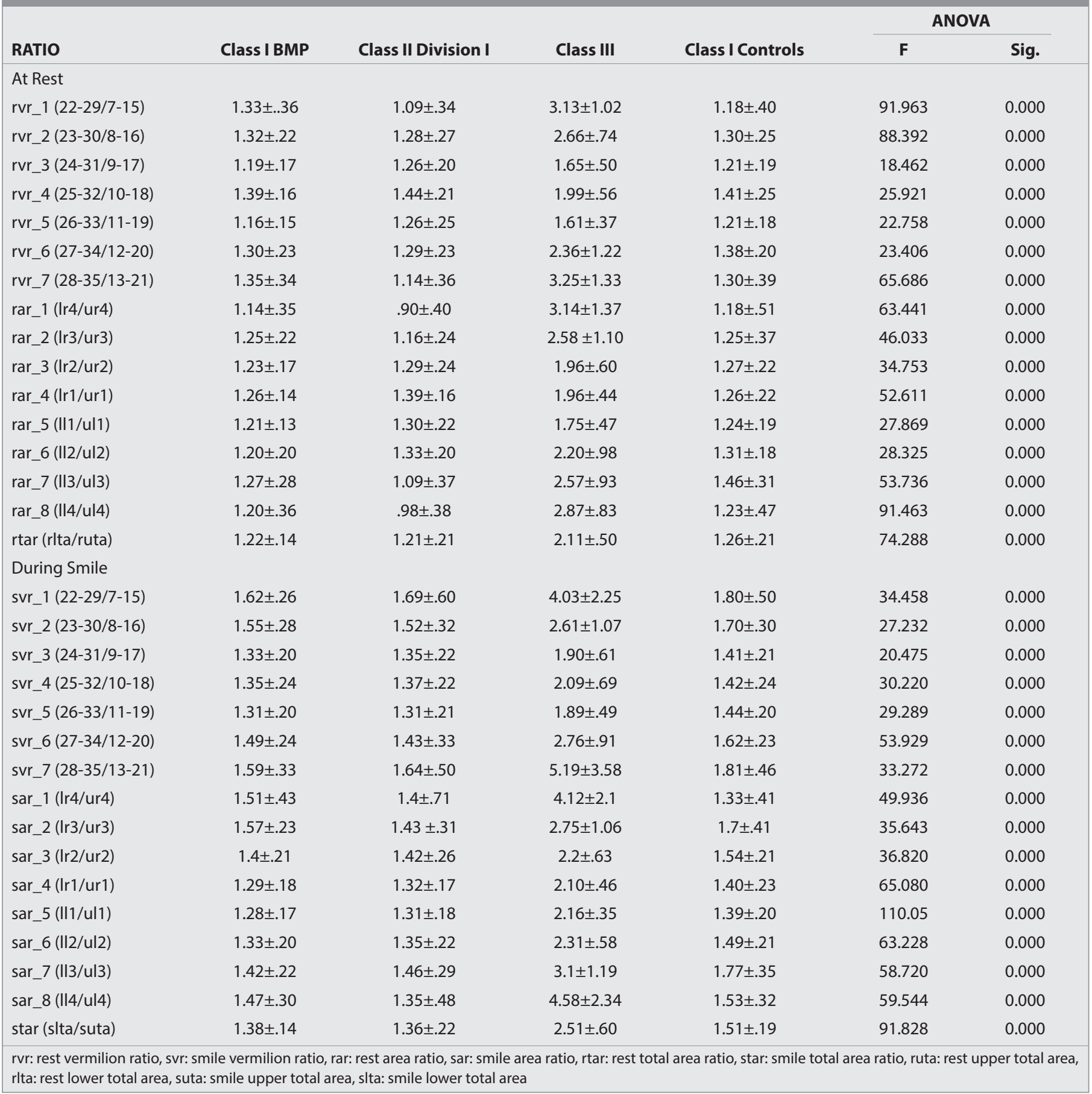

vermilion heights as compared to Class III and Class I controls. This agrees with a previous observation by Nety Trisnawaty et al. (5). The probable reason for this could be that flared incisors have a tendency to roll the upper and lower lips out, exposing more of the mucocutaneous lip and increasing the vermilion height and lip area (7).

Skeletal Class II individuals with increased overjet have been reported to have upper lips more protrusive than lower by Chihiro Tanikawa et al. (4). They hypothesized that the upper lip may be looked upon as thicker (or vertically longer) because the upper-lip vermilion receives, in theory, less vertical pressure from the lower-lip vermilion (4). The present study also supports the above hypothesis as a significant increase in the size of the upper lip was observed in skeletal Class II cases (Table 2).

On computing the upper-to-lower-lip ratios for all the four groups, our study revealed that the ratios were significantly increased in the skeletal Class III for both the vermilion height and lip area at rest and during smile. The increase in ratio was three times greater at rest and four times greater during smile at extreme right and left corners of the mouth when compared with Class I (Figure 4). This is similar to the observation by Rafiqul Islam et al. who attributed an increased lip ratio to the everted 


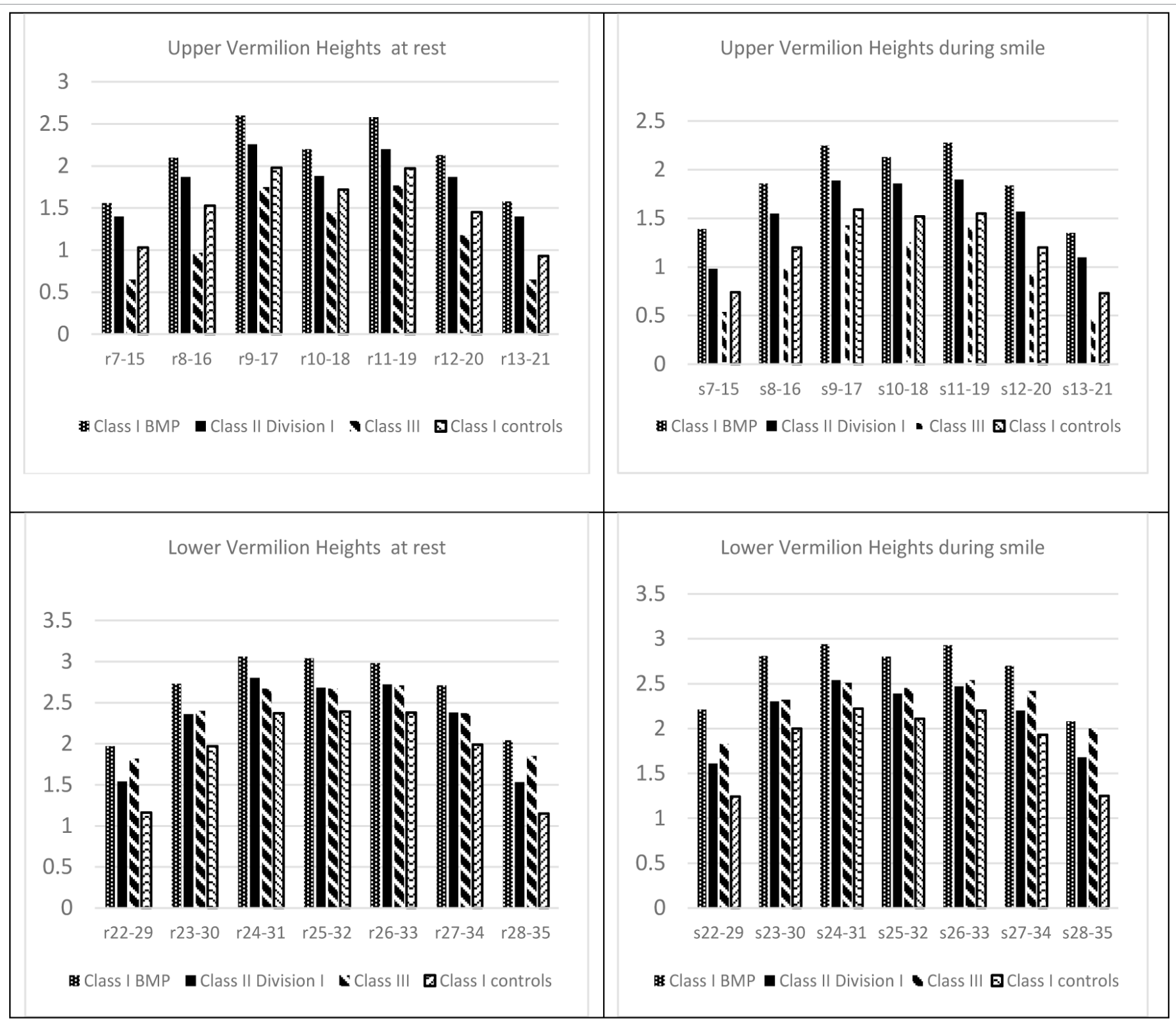

Figure 3. Comparison of the upper- and lower-segment-wise vermilion heights at rest and during smile r: rest, s: smile

lower lip seen in cases of reverse overbite and skeletal Class III malocclusions; this would increase the lower-lip area resulting in loss of the upper and lower-lip balance (17). However, the results of our investigation do not show any significant change in the lower lip of Class III group when compared to Class I controls. The upper lip on the other hand showed a significant decrease in the upper-lip area and vermilion height in Class III group, which resulted in the increased lip ratios. In individuals with skeletal Class III malocclusion, the lower-lip vermilion, positioned more forward than its opponent, pushes the upper-lip vermilion backward, and a part of the upper-lip vermilion rolls inwards (4). Previous investigations reporting retrusive upper lips and protrusive lower lips in skeletal Class III malocclusions have all evaluated lips in the lateral/profile view only $(4,20)$.

A closer look at Figure 4 revealed interesting features. Both the vermilion height- and segment-wise lip-area ratios increased progressively from the midline to the extreme right and left corners of the mouth for the Class III group. Such a pattern was not observed for the Class I or Class II Div 1 subjects. The vermilion height ratios at the mouth corners were three times greater than the midline at rest and four times greater during smile in the Class III group. This was due to the fact that the observed decrease in the upper vermilion height was more towards the corners of the mouth.

\section{Clinical Implications}

The close association between sagittal dentoskeletal discrepancies and the vermilion height/lip area is obvious from the findings of this study. Considering the trend toward preferences for increased lip fullness, techniques to gain the arch length and non-extraction treatments are catching on. The use of expansion appliances, lip bumper, lingual arch, the Schwarz plate, and various molar distalization appliances are now supplanting premo- 


\section{At Rest}

Vermilion Height Ratios

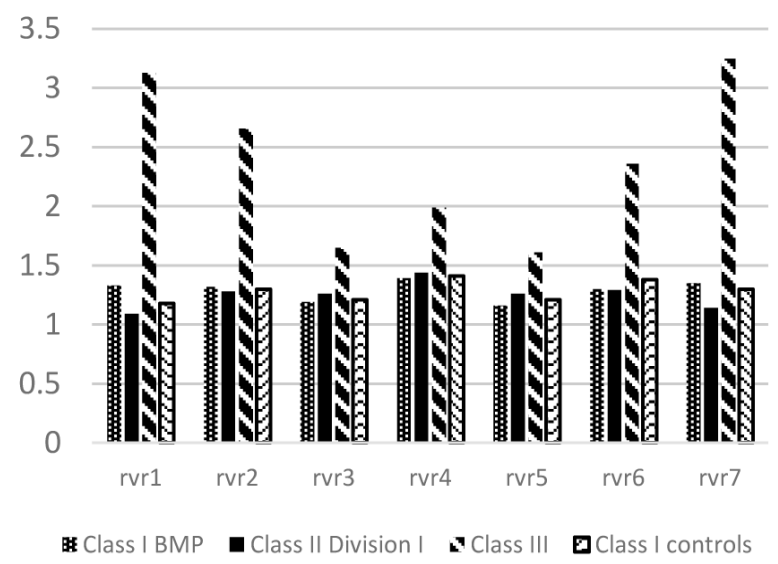

Segment wise Area Ratio

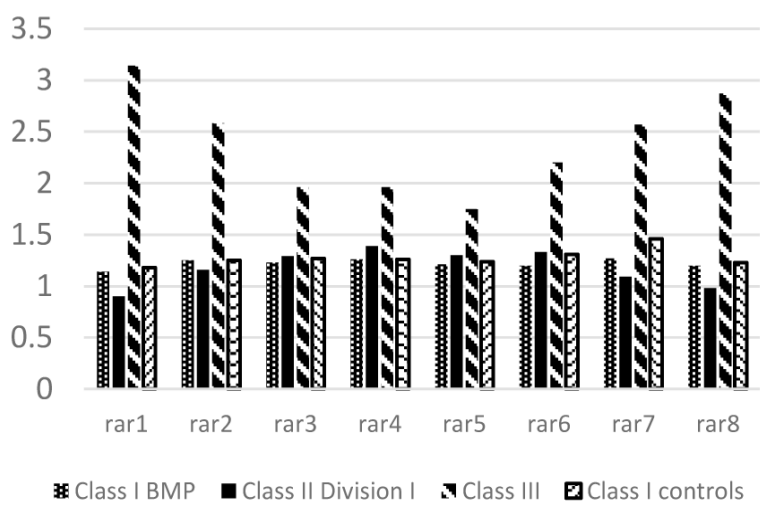

Total Area Ratio

2.5

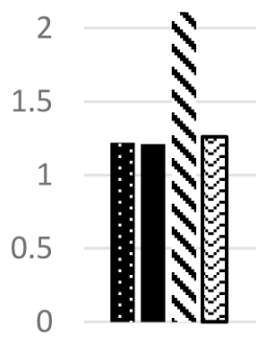

-Class | BMP \#Class || Division I "Class III ‘Class | controls

\section{During Smile}

Vermilion Height Ratios

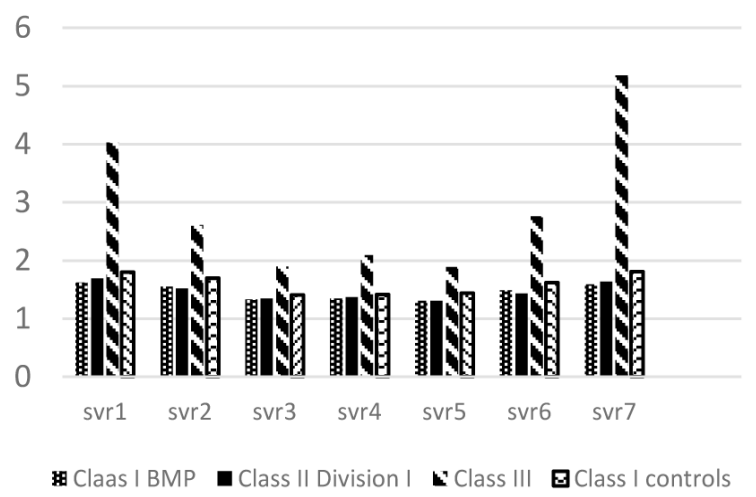

Segment Wise Area Ratio

5

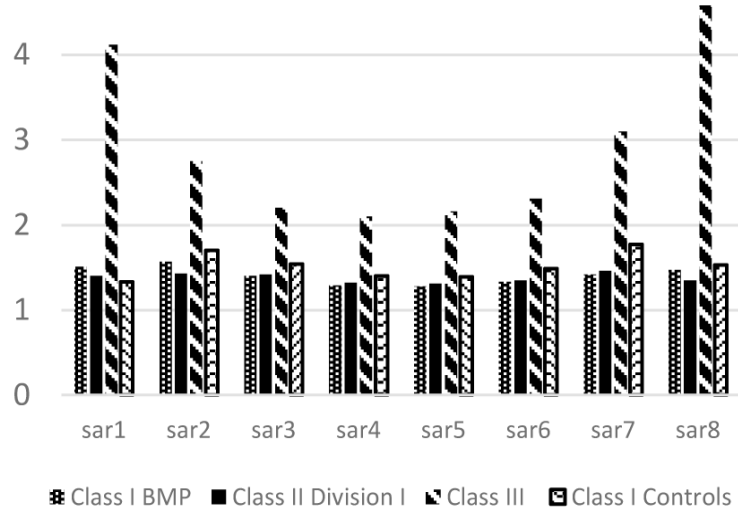

Total Area Ratio

3

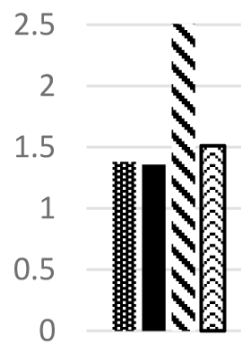

\# Class I BMP

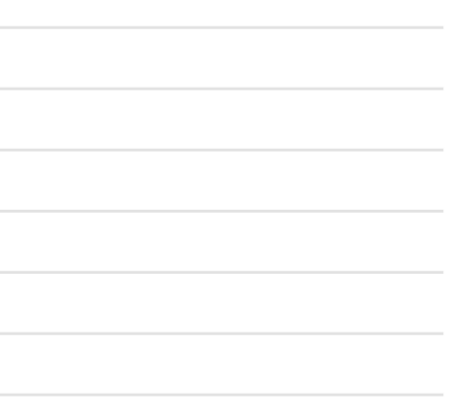

Class II Division I "Class III $\quad$ Class I controls 
lar extractions (8). As morphologic characteristics of lips reflect underlying sagittal skeletal discrepancies, they could also serve as additional diagnostic indicators during a clinical examination of the face.

\section{Limitation of the Study}

The effect of vertical skeletal discrepancies and Class II Division 2 malocclusion on frontal lip morphology was not included. This needs to be evaluated in further studies. Only females were included, considering the effect of sexual dimorphism on the lip morphology. Males may also have similar lip ratios, which were not evaluated in this study.

\section{CONCLUSION}

- Morphologic characteristics of lips showed significant differences among skeletal Class I, Class II, and Class III malocclusions at rest and during smile.

- Skeletal Class III cases displayed a significantly smaller upper-lip area, as compared to the Class I BMP, Class II Div 1, and Class I N group.

86 - Class I BMP and Class II Div 1 displayed significantly larger upper and lower lip areas.

- Ratios of the upper-to-lower-lip areas and vermilion heights showed significantly higher values for skeletal Class III. The ratios displayed an increasing trend from the midline to the corners of the mouth.

No statistically significant differences were observed in the lip-area ratios and vermilion height ratios for Class I BMP, Class II Div 1, and Class I N.

Ethics Committee Approval: Ethics committee approval was received for this study from the Institutional Ethics Committee of Government Dental College (IEC no: 45/2014/DCC).

Informed Consent: Written informed consent was obtained from the patients who participated in this study.

Peer-review: Externally peer-reviewed.

Author Contributions: Concept - S.Sundareswaran; Design - S.Sundareswaran; Data Collection and/or Processing - A.J., S.Srinivas; Analysis and/or Interpretation -A.J., S.Sundareswaran; Literature Search - A.J., S.Srinivas; Writing Manuscript - A.J., S.Sundareswaran, S.Srinivas; Critical Review - S.Sundareswaran.

Conflict of Interest: The authors have no conflict of interest to declare.

Financial Disclosure: The authors declared that this study has received no financial support.

\section{REFERENCES}

1 Van der Geld P, Oosterveld P, Schols J, Kuijpers-Jagtman AM. Smile line assessment comparing quantitative measurement and visu- al estimation. Am J Orthop Dentofac Orthop 2011; 139: 174-80. [CrossRef]

2. Cao L, Zhang K, Bai D, Jing Y, Tian Y, Guo Y. Effect of maxillary incisor labiolingual inclination and anteroposterior position on smiling profile esthetics. Angle Orthod 2011; 81: 121-9. [CrossRef]

3. Peck H, Peck S. A concept of facial esthetics. Angle Orthod 1970; 40: 284-318.

4. Tanikawa C, Nakamura K, Yagi M, Takada K. Lip Vermilion Profile Patterns and Corresponding Dentoskeletal Forms in Female Adults. Angle Orthod 2009; 79: 849-58. [CrossRef]

5. Trisnawaty N, loi H, Kitahara T, Suzuki A, Takahashi I. Effects of extraction of four premolars on vermilion height and lip area in patients with bimaxillary protrusion. Eur J Orthod 2013; 35: 521-8. [CrossRef]

6. Sforza C, Laino A, D'Alessio R, Grandi G, Tartaglia GM, Ferrario VF. Soft-tissue facial characteristics of attractive and normal adolescent boys and girls. Angle Orthod 2008; 78: 799-807. [CrossRef]

7. McNamara L, McNamara JA, Ackerman MB, Baccetti T. Hard- and soft-tissue contributions to the esthetics of the posed smile in growing patients seeking orthodontic treatment. Am J Orthop Dentofac Orthop 2008; 133: 491-9. [CrossRef]

8. Turley PK. Evolution of esthetic considerations in orthodontics. Am J Orthop Dentofac Orthop 2015; 148: 374-9. [CrossRef]

9. loi H, Kang S, Shimomura T, Kim S, Park S, Son W, Takahashi I. Effects of vermilion height on lip esthetics in Japanese and Korean orthodontists and orthodontic patients. Angle Orthod 2014; 84: 239-45 [CrossRef]

10. Sundareswaran S, Kumar V. Reliability of Beta angle in assessing true anteroposterior apical base discrepancy in different growth patterns. J Nat Sc Biol Med 2015; 6: 125-30. [CrossRef]

11. Islam R, Kitahara $T$, Naher $L$, Hara A, Nakasima A. Lip morphological changes in orthodontic treatment. Class II division 1: malocclusion and normal occlusion at rest and on smiling. Angle Orthod 2009; 79: 256-64. [CrossRef]

12. Ferrario VF, Sforza C, Pizzini G, Vogel G, Miani A. Sexual dimorphism in the human face assessed by euclidean distance matrix analysis. J Anat 1993; 183: 593-600.

13. Ferrario VF, Sforza C, Schmitz JH, Miani A, Taroni G. Fourier analysis of human soft tissue facial shape: sex differences in normal adults. J Anat 1995; 187: 593-602.

14. Miron $\mathrm{H}$, Calderon S, Allon D. Upper lip changes and gingival exposure on smiling: vertical dimension analysis. Am J Orthop Dentofacial Orthop 2012; 141: 87-93.[CrossRef]

15. Mamandras AH. Linear changes of the maxillary and mandibular lips. Am J Orthop Dentofac Orthop 1988; 94: 405-10. [CrossRef]

16. Nanda RS, Meng H, Kapila S, Goorhuis J. Growth changes in the soft tissue facial profile. Angle Orthod 1990; 60: 177-90.

17. Islam R, Kitahara T, Naher L, Hara A, Nakata S. Lip Morphology Changes Following Orthognathic Surgery for Class III Malocclusion. Angle Orthod 2010; 80: 344-53. [CrossRef]

18. Malkoç S, Demir A, Uysal T, Canbuldu N. Angular photogrammetric analysis of the soft tissue facial profile of Turkish adults. Eur J Orthod 2009; 31: 174-9. [CrossRef]

19. Zhang X, Hans MG, Graham G, Kirchner HL, Redline S. Correlations between cephalometric and facial photographic measurements of craniofacial form. Am J Orthop Dentofac Orthop 2007; 131: 67-71. [CrossRef]

20. Joshi M, Wu LP, Maharjan S, Regmi MR. Sagittal lip positions in different skeletal malocclusions: a cephalometric analysis. Prog Orthod 2015; 16: 8. [CrossRef] 